\title{
The effect of dissipation on quantum transmission resonance
}

\author{
Kohkichi Konno, ${ }^{1}$ Munehiro Nishida, ${ }^{2}$ Satoshi Tanda,, , $*$ and Noriyuki Hatakenaka ${ }^{3}$ \\ ${ }^{1}$ Department of Applied Physics, Hokkaido University, Sapporo 060-8628, Japan. \\ ${ }^{2}$ Graduate School of Advanced Sciences of Matter, \\ Hiroshima University, Higashi-Hiroshima 739-8530, Japan. \\ ${ }^{3}$ Graduate School of Integrated Arts and Sciences, \\ Hiroshima University, Higashi-Hiroshima 739-8521, Japan.
}

(Dated: November 14, 2018)

\begin{abstract}
Quantum transmissions of a free particle passing through a rectangular potential barrier with dissipation are studied using a path decomposition technique. Dissipative processes strongly suppress the transmission probability at resonance just above the barrier resulting in an unexpected reduction of the mean traversal time through the potential barrier.
\end{abstract}

\section{INTRODUCTION}

Quantum mechanics successfully describes physical processes at the microscopic scale and sometimes exhibits unique counter-intuitive phenomena. One such example is quantum-mechanical tunneling that cannot be explained in terms of classical mechanics. In search of an applicability for quantum mechanics on a macroscopic scale [1], the effect of dissipation on quantum tunneling has been studied in respect to systems such as superconducting devices [2, 3, 4] because dissipation is inherent and inevitable in macroscopic scale. The observation of quantum-tunneling-rate reduction was a first important result to be associated with quantum mechanics on a macroscopic scale.

Quantum tunneling, however, is not the only intrinsic feature of quantum mechanics. Another unconventional example in a classical sense is quantum reflection. Suppose that there is a particle incident to a potential barrier, which has a height slightly lower than the particle's energy, as shown in Fig. 1(a). According to classical mechanics, the particle overcomes the barrier and is never reflected back. In quantum mechanics, there is the possibility that the particle cannot go over the barrier. This leads to a remarkable phenomenon called a transmission resonance. That is due to multiple quantum reflections between potential boundaries. In other words, the resonance occurs due to interference associated with the back-and-forth motions of the particle. Quantum tunneling only shows its quantum-mechanical feature at the moment of the tunneling event, while the interference requires for a certain definite period to retain quantum coherence based on the quantum-mechanical superposition of states. Therefore, quantum interference appears a more convincing quantum-mechanical effect than does quantum tunneling. Indeed, the effect of dissipation on the quantum-mechanical superposition of macroscopically distinguishable states, so-called Schrödinger's cat in a fundamental problem of quantum mechanics,

*tanda@eng.hokudai.ac.jp has been studied along these lines in respect to superconducting nanodevices [5, 6] for example. Recently, this cat state has been utilized as a building block for a quantum computer in quantum information science. Furthermore, direct observations of matter wave interference revealed that inevitable sources of dissipation for large molecules, e.g. collisions to external molecules [7] or internal vibrations resulting in thermal photon radiations [8], play a key role for the quantum-to-classical transition of "free" particle. Even the gravitational waves were suggested to become a source of decoherence of matter waves 9]. Thus, macroscopic transmission resonance originated from quantum interference of macroscopic object also provides an alternative platform to that of quantum tunneling for testing the validity of quantum mechanics on a macroscopic scale.

The transmission of a particle through a rectangular potential barrier in the absence of dissipation has been well studied within the framework of Schrödinger's wave mechanics at the textbook level. A simple extension for incorporating dissipation in that framework was made by Cai et al.[10] to deal with the problem that an electron propagates above a quantum well with dissipation due to the electron-optical-phonon interaction. They succeeded in revealing the electron-capture process in a quantum well that involves a loss of electron energy via phonon emission. However, in order to investigate the problem addressed here, we need an alternative approach in which the influence of the particle motion on the environment is also included. In particular, our interest is directed to the transmission resonance formed just above the potential barrier where the Wentzel-Kramers-Brillouin (WKB) method adopted in previous studies [11, 12] breaks down.

In this Letter, we employ a path decomposition expansion method [13, 14, 15, 16] based on the path-integral approach, and develop it to incorporate dissipative processes. Then we discuss the effect of dissipation on quantum transmission resonance. Since the resonance may be attributed to the back-and-forth motions of a particle between the potential boundaries, as pointed out by Bohm in his seminal book [17], the particle will stay in region II for a longer time at resonance. Thus the resonance could be characterized by the time spent in the potential barrier 
(a)

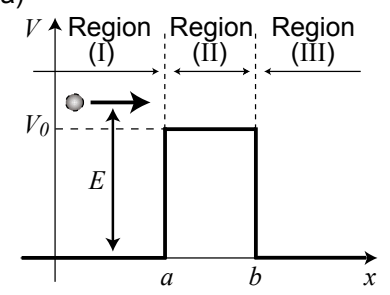

(b)

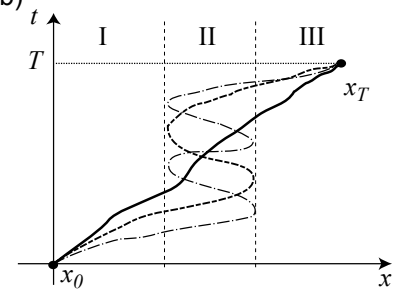

(c)

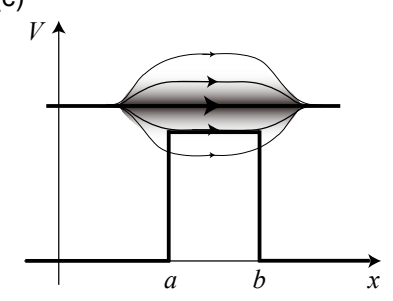

(d)

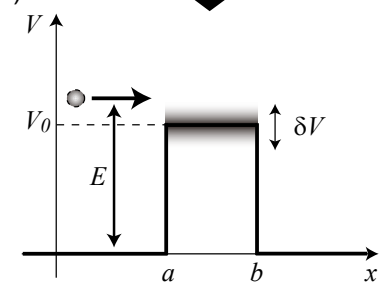

FIG. 1: Schematic diagrams of (a) three decomposed regions for a rectangular potential barrier, (b) typical possible paths, (c) uncertainty of energy, and (d) uncertainty of potential height, where the diagram (c) is equivalent to (d).

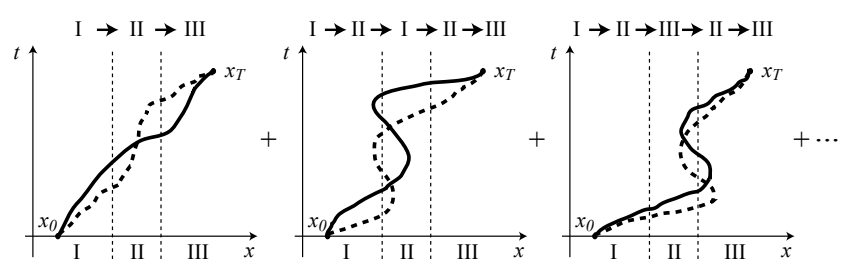

FIG. 2: Illustration of the path decomposition technique. Several groups of summations of all possible paths are shown (see also text). Each group is classified according to which regions the paths have passed over in terms of temporal development. Two examples of the possible paths are shown by solid and dashed curves in each figure.

region, i.e., the traversal time. Therefore, we investigate dissipative quantum transmission resonance in terms of traversal time on the basis of Bohm's interpretation.

This Letter is organized as follows. In Section [I] we formulate the transmission probability through a rectangular potential barrier with dissipation by using a path decomposition technique. We also perform numerical calculations of the transmission probabilities. Then we will find the non-uniform reduction of transmission probability even though we assume an energy-independent damping processes. In Section III. We introduce the traversal time under dissipation to explain the unexpected reduction on the basis of Bohm's interpretation. We also provide further evidence for our interpretation by using traversal time distribution, which results in an unexpected shortened mean traversal time. In Section [V] we provide a summary and propose a possible experimental setup.

\section{TRANSMISSION PROBABILITY IN THE PRESENCE OF DISSIPATION}

\section{A. Analytical description of transmission probability}

We briefly review a path decomposition expansion developed by Auerbach and Kivelson [13]. This enables us to deal with quantum transmission in terms of the path integral approach. In the path decomposition technique, the summation of possible paths is decomposed into certain groups as shown in Fig. 2. The first group is composed of all possible paths that pass straight over regions I, II, and III in that order. The next group includes all paths that go from region I to region II, then return to region I once, and go from region II to III. In the same way, one can consider all other groups. Thus, the total summation of paths is given by an infinite series of groups as shown in Fig. 2. Each group is completely expressed by the propagators $K^{(\mathrm{I})}\left(x^{\prime}, x\right), K^{(\mathrm{II})}\left(x^{\prime}, x\right)$ and $K^{(\mathrm{III})}\left(x^{\prime}, x\right)$ defined in the restricted regions I, II and III, respectively. Since all possible paths are taken for summation, no approximation is used in this technique, the point of which prevails against the WKB method. The propagator $K\left(x_{T}, x_{0} ; T\right)$ from $x_{0}(<a)$ at $t=0$ to $x_{T}(>b)$ at $t=T$ is then decomposed as [13]

$$
\begin{array}{rl}
i K\left(x_{T}, x_{0} ; T\right) & \\
=\int_{0}^{T} & d t_{1} \int_{0}^{T-t_{1}} d t_{2} i K^{(\mathrm{I})}\left(a, x_{0} ; t_{1}\right) \Sigma_{x}^{a}\left(i K^{(\mathrm{II})}\left(b, x ; t_{2}\right)\right) \\
& \times \Sigma_{x}^{b}\left(i K^{(\mathrm{III})}\left(x_{T}, x ; T-t_{1}-t_{2}\right)\right) \\
+ & \int_{0}^{T} d t_{1} \int_{0}^{T-t_{1}} d t_{2} \int_{0}^{T-t_{1}-t_{2}} d t_{3} \int_{0}^{T-t_{1}-t_{2}-t_{3}} d t_{4} \\
& \times i K^{(\mathrm{I})}\left(a, x_{0} ; t_{1}\right) \Sigma_{x}^{a}\left(i K^{(\mathrm{II})}\left(a, x ; t_{2}\right)\right) \\
& \times \Sigma_{x}^{a}\left(i K^{(\mathrm{I})}\left(a, x ; t_{3}\right)\right) \Sigma_{x}^{a}\left(i K^{(\mathrm{II})}\left(b, x ; t_{4}\right)\right) \\
& \times \Sigma_{x}^{b}\left(i K^{(\mathrm{III})}\left(x_{T}, x ; T-t_{1}-t_{2}-t_{3}-t_{4}\right)\right) \\
+ & \cdots
\end{array}
$$

where $\Sigma_{x}^{a}$ denotes a derivative operator defined by

$$
\left.\Sigma_{x}^{a}\left(i K^{(\mathrm{II})}(b, x ; t)\right) \equiv \epsilon_{a b} \frac{\hbar}{2 m} \frac{\partial}{\partial x}\left(i K^{(\mathrm{II})}(b, x ; t)\right)\right|_{x=a} .
$$

Here, $m$ is the particle mass, and

$$
\epsilon_{a b} \equiv\left\{\begin{array}{cc}
1 & (a<b) \\
-1 & (a>b)
\end{array}\right.
$$

(see Ref. [13] for details).

In the absence of dissipation, the propagator $K^{(\mathrm{II})}$ in region II is expressed as [18]

$K^{(\mathrm{II})}\left(x^{\prime}, x ; t\right)$ 


$$
\begin{aligned}
& =\sqrt{\frac{m}{2 \pi i \hbar t}} \sum_{n=-\infty}^{\infty}\left\{\exp \left[\frac{i m\left(2 n d+x^{\prime}-x\right)^{2}}{2 \hbar t}\right]\right. \\
& \left.-\exp \left[\frac{i m\left((2 n+1) d-\left(x^{\prime}-b\right)-(x-a)\right)^{2}}{2 \hbar t}\right]\right\}(4)
\end{aligned}
$$

where $d$ is defined by $d=b-a$, and $n$ characterizes different classical paths in each term. The first term is composed of all paths with even numbers of reflections at the boundaries of $x=a$ and $x=b$, and the second term corresponds to the odd numbers of reflections. Paths that start to move in a negative direction in relation to the initial position are characterized by negative $n$. Equation (4) indicates that the propagator in region II is essentially expressed by the summation of a free particle's propagator;

$$
K_{0}\left(x^{\prime}, t ; x, 0\right)=\sqrt{\frac{m}{2 \pi i \hbar t}} \exp \left[\frac{i m\left(x^{\prime}-x\right)^{2}}{2 \hbar t}\right] .
$$

Now let us consider the propagator $K_{\mathrm{D}}^{(\mathrm{II})}$ in the presence of dissipation. Dissipation in quantum mechanics has long been discussed since it cannot be included as a form of analytical mechanics. It remains unresolved. However, several aspects such as dissipative quantum tunneling and quantum Brownian motion have been presented. Here we employ a phenomenological model successfully introduced by Caldeira and Leggett [19], to describe dissipation in a study of quantum Brownian motion of a particle in harmonic potential. They modeled an environment as a set of a huge number of harmonic oscillators that produces a classical equation of motion with dissipation. We apply their model to a free particle coupled to the environment.

According to their model, the effect of the propagator $K_{\mathrm{D}}^{(\mathrm{II})}$ is included in the expression for the time evolution of the system of interest coupled to environment described by

$$
\rho(x, y, t)=\int d x_{\mathrm{i}} d y_{\mathrm{i}} J\left(x, y, t ; x_{\mathrm{i}}, y_{\mathrm{i}}, 0\right) \rho\left(x_{\mathrm{i}}, y_{\mathrm{i}}, 0\right),
$$

where $J\left(x, y, t ; x^{\prime}, y^{\prime}, 0\right)$ is the propagator for the density matrix $\rho(x, y, t)=\langle x \mid \psi(t)\rangle\langle\psi(t) \mid y\rangle$ of the free particle, and the autocorrelation of stochastic force $F_{\mathrm{cl}}(\tau)$

$$
\left\langle F_{\mathrm{cl}}(\tau) F_{\mathrm{cl}}(s)\right\rangle=2 \eta k_{\mathrm{B}} T^{\prime} \delta(\tau-s)
$$

is imposed on it. Indeed, in the absence of dissipation, the above expression for a free particle includes the free particle's propagator $K_{0}$ as

$\rho(x, y, t)=\int d x_{\mathrm{i}} d y_{\mathrm{i}} K_{0}^{*}\left(y, t ; y_{\mathrm{i}}, 0\right) K_{0}\left(x, t ; x_{\mathrm{i}}, 0\right) \rho\left(x_{\mathrm{i}}, y_{\mathrm{i}}, 0\right)$.

In particular, when the initial state is given by $\langle x \mid \psi(0)\rangle=$ $\delta\left(x-x_{0}\right)$, we simply have

$$
\rho(x, x, t)=K_{0}^{*}\left(x, t ; x_{0}, 0\right) K_{0}\left(x, t ; x_{0}, 0\right)=\frac{m}{2 \pi i \hbar t} .
$$

In the case of a free particle coupled to a set of harmonic oscillators, with $\langle x \mid \psi(0)\rangle=\delta\left(x-x_{0}\right)$, the density matrix is given by

$$
\rho(x, x, t)=\frac{m}{2 \pi \hbar t} f(t),
$$

with

$$
f(t) \equiv \frac{\sigma t e^{\gamma t}}{\sinh \sigma t},
$$

where $\gamma$ is a relaxation rate, and $\sigma$ is defined by $\sigma \equiv$ $\sqrt{\gamma^{2}+(4 \gamma \Omega / \pi)^{2}}$. Here $\Omega$ is the cutoff frequency for the frequency distribution of harmonic oscillators. The function $f(t)$ can be obtained by integrating the degrees of freedom of the environment and, therefore, includes the effect of the associated harmonic oscillators. Since the cutoff frequency $\Omega$ is sufficiently large compared with $\gamma$, the function $f(t)$ becomes a monotonically decreasing function, which decays exponentially, i.e., $f(t) \propto e^{-(\sigma-\gamma) t}$. It should also be noted that $f(0)=1$ and $f(t)>0$ for $t>0$. In the limit of $\gamma \rightarrow 0$, since $f(t) \rightarrow 1$, we retrieve the result for the nondissipative case $\left|K_{0}\left(x, t ; x_{0}, 0\right)\right|^{2}=m / 2 \pi \hbar t$, where $K_{0}$ is the free particle's propagator shown in Eq. (5). Therefore, we can consider the propagator for the dissipative case to be effectively expressed as

$$
K_{\mathrm{D}}\left(x, t ; x_{0}, 0\right)=\sqrt{f(t)} K_{0}\left(x, t ; x_{0}, 0\right),
$$

which gives the same result as Eq. (10).

The propagator in region II is obtained by summing up the free particle's propagators along possible classical paths. Thus, we obtain the effective propagator in region II with dissipation as

$$
K_{\mathrm{D}}^{(\mathrm{II})}\left(x^{\prime}, x ; t\right)=\sqrt{f(t)} K^{(\mathrm{II})}\left(x^{\prime}, x ; t\right) .
$$

The full propagator, taking account of the dissipative effect, is given by

$$
\begin{array}{rl}
i K_{\mathrm{D}}\left(x_{T},\right. & \left.x_{0} ; T\right) \\
=\int_{0}^{T} & d t_{1} \int_{0}^{T-t_{1}} d t_{2} \sqrt{f\left(t_{2}\right)} i K^{(\mathrm{I})}\left(a, x_{0} ; t_{1}\right) \\
& \times \Sigma_{x}^{a}\left(i K^{(\mathrm{II})}\left(b, x ; t_{2}\right)\right) \\
& \times \Sigma_{x}^{b}\left(i K^{(\mathrm{III})}\left(x_{T}, x ; T-t_{1}-t_{2}\right)\right) \\
+ & \int_{0}^{T} d t_{1} \int_{0}^{T-t_{1}} d t_{2} \int_{0}^{T-t_{1}-t_{2}} d t_{3} \int_{0}^{T-t_{1}-t_{2}-t_{3}} d t_{4} \\
& \times \sqrt{f\left(t_{2}+t_{4}\right)} i K^{(\mathrm{I})}\left(a, x_{0} ; t_{1}\right) \Sigma_{x}^{a}\left(i K^{(\mathrm{II})}\left(a, x ; t_{2}\right)\right) \\
& \times \Sigma_{x}^{a}\left(i K^{(\mathrm{I})}\left(a, x ; t_{3}\right)\right) \Sigma_{x}^{a}\left(i K^{(\mathrm{II})}\left(b, x ; t_{4}\right)\right) \\
& \times \Sigma_{x}^{b}\left(i K^{(\mathrm{III})}\left(x_{T}, x ; T-t_{1}-t_{2}-t_{3}-t_{4}\right)\right) \\
+ & \cdots,
\end{array}
$$

where since $f(t)$ behaves as an exponentially decaying function, we have used the approximation $f(t) f\left(t^{\prime}\right) \approx$ $f\left(t+t^{\prime}\right)$, whose iterative use leads to $\prod_{i} f\left(t_{i}\right) \approx f\left(\sum_{i} t_{i}\right)$. 
To convert Eq. (14) into an energy representation, we perform a Fourier transform of the propagator as

$$
G_{\mathrm{D}}\left(x_{T}, x_{0} ; E\right) \equiv i \int_{0}^{\infty} d T K_{\mathrm{D}}\left(x_{T}, x_{0} ; T\right) e^{i E T / \hbar} .
$$

Utilizing the expression

$$
\begin{aligned}
& \sqrt{f\left(\Sigma_{i} t_{i}\right)} \\
& =\int_{0}^{\infty} d \tau \sqrt{f(\tau)} \delta\left(\tau-\Sigma_{i} t_{i}\right) \\
& =\int_{0}^{\infty} d \tau \sqrt{f(\tau)} \frac{1}{2 \pi} \int_{-\infty}^{\infty} d \omega e^{-i \omega\left(\tau-\Sigma_{i} t_{i}\right)}
\end{aligned}
$$

we finally obtain the Green function including the dissipative effect as

$$
G_{\mathrm{D}}\left(x_{T}, x_{0} ; E\right)=w_{\mathrm{D}}\left(E, V_{0}\right) G_{0}\left(x_{T}, x_{0} ; E\right),
$$

where $G_{0}\left(x_{T}, x_{0}\right)$ is the Green function when the barrier is absent, and the transmission amplitude $w_{\mathrm{D}}\left(E, V_{0}\right)$ is given by

$$
\begin{aligned}
& w_{\mathrm{D}}\left(E, V_{0}\right) \\
& =\int_{0}^{\infty} d \tau \sqrt{f(\tau)} \int_{-\infty}^{\infty} \frac{d \omega}{2 \pi} e^{-i \omega \tau} w\left(E, V_{0}-\hbar \omega\right) .
\end{aligned}
$$

Here, $w\left(E, V_{0}\right)$ is the transmission amplitude in the absence of dissipation

$$
w\left(E, V_{0}\right)=-\frac{2 i k \kappa e^{-i k d}}{\left(k^{2}+\kappa^{2}\right) \sin \kappa d+2 i k \kappa \cos \kappa d},
$$

where

$$
k \equiv \frac{\sqrt{2 m E}}{\hbar}, \quad \kappa \equiv \frac{\sqrt{2 m\left(E-V_{0}\right)}}{\hbar} .
$$

The paths in an energy representation are introduced by the potential deviations in terms of an energy quantum $\hbar \omega$ as shown in Fig. 1 (c) and (d). Therefore, the transmission probability including the effect of dissipation is given by $\left|w_{\mathrm{D}}\left(E, V_{0}\right)\right|^{2}$. Equations (17) and (18) are our main result.

\section{B. Numerical estimates of transmission probabilities}

In our numerical calculations, we restrict ourselves to a constant $\gamma$ damping for any $E$ even though the relaxation rate $\gamma$ may depend on the incident energy $E$ of the particle in general. Figure 3 shows the transmission probabilities for different $\gamma$ values. Two dimensionless parameters characterize the transmission probability, i.e., the particle energy normalized by the potential height, $E / V_{0}$, and the potential width normalized by the typical scale of length, $d / \lambda_{0}$, where $\lambda_{0} \equiv \hbar / \sqrt{2 m V_{0}}$. The dashed curve shows the transmission probabilities for a non-dissipative

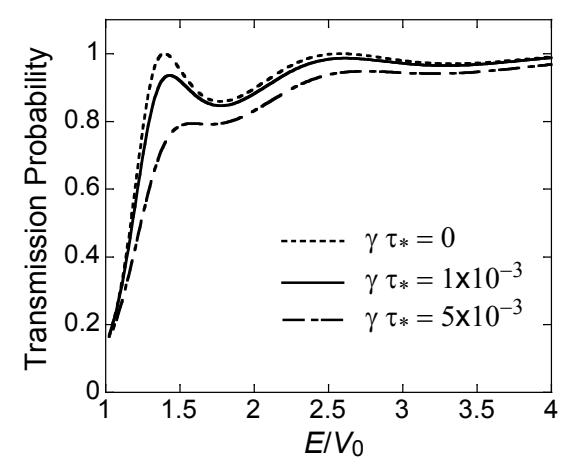

FIG. 3: Transmission probabilities as a function of $E / V_{0}$. $\gamma \tau_{*}=5 \times 10^{-3}, 1 \times 10^{-3}$ and 0 are plotted, where $\tau_{*} \equiv$ $\sqrt{m d^{2} / 2 V_{0}}$. In the calculations, we adopted $d / \lambda_{0}=5$ and $\Omega \tau_{*}=100$.

case. The oscillatory structure is caused by transmission resonance, which arises as a result of quantum reflection at the barrier edges. From Eq. (19), perfect transmission occurs whenever the barrier contains an integer number of a half wavelength, i.e., $\kappa d=n \pi(n=1,2, \cdots)$.

In the presence of dissipation, the transmission probabilities are greatly suppressed. The blurred oscillatory structure implies that the suppression is not uniform with respect to energy, even for the energy-independent relaxation rate $\gamma$. Indeed, significant suppression occurs around the resonance conditions.

\section{TRAVERSAL TIME THROUGH BARRIER}

\section{A. Traversal time and transmission probability}

In a classical description transmission resonance can be attributed to the back-and-forth motion of a particle between the edges of the potential barrier. This will lead to a longer stay in region II, equivalent to a longer traversal time. Hence we can investigate the transmission probabilities in the presence of dissipation in terms of the traversal time through region II.

In terms of the path integral formalism [14, 15, 16], the traversal time has a distribution, because all possible paths are considered and a certain weight is assigned to each path in the path integral. According to Fertig [16], the probability amplitude of a particle spending time $\tau$ in region II is defined by

$$
F(\tau) \equiv \frac{\sum_{\text {path:C }} e^{i S(C) / \hbar} \delta\left(\tau-\tau_{\mathrm{II}}(C)\right)}{\sum_{\text {path:C}} e^{i S(C) / \hbar}},
$$

where $C$ denotes a path, $S$ denotes the action, and $\tau_{\mathrm{II}}(C)$ is the traversal time through region II along path $C$. The denominator in Eq. (21) is by definition equivalent to the propagator $K\left(x_{T}, x_{0} ; T\right)$. The delta function in the numerator extracts the paths with traversal time $\tau$. In 
the following discussion, we assume a constant energy state within the limits of $x_{0} \rightarrow-\infty$ and $x_{T} \rightarrow \infty$.

First, we investigate the relationship between the transmission resonance and the traversal time in the absence of dissipation. The mean traversal time $\langle\tau\rangle$ is defined by

$$
\langle\tau\rangle \equiv \int_{0}^{\infty} \tau F(\tau) d \tau
$$

In the case of a rectangular potential barrier it is given by [16],

$$
\begin{aligned}
\langle\tau\rangle=\frac{m}{\hbar} & \frac{2 k}{\kappa} \frac{A \kappa d-B \sin \kappa d \cos \kappa d}{B^{2} \sin ^{2} \kappa d+4 k^{2} \kappa^{2}} \\
& +i \frac{m}{\hbar} \frac{B \kappa d \cos \kappa d-A \sin \kappa d}{B^{2} \sin ^{2} \kappa d+4 k^{2} \kappa^{2}} \frac{B}{\kappa^{2}} \sin \kappa d,
\end{aligned}
$$

where

$$
A \equiv k^{2}+\kappa^{2}, \quad B \equiv k^{2}-\kappa^{2} .
$$

Under the resonance (the constructive interference) conditions $\kappa d=n \pi(n=1,2, \cdots)$, Equation (23) is reduced to the expression

$$
\langle\tau\rangle=\frac{m d A}{2 \hbar k \kappa^{2}} .
$$

Using the inequality

$$
\frac{\alpha+\beta}{2} \geq \sqrt{\alpha \beta} \quad(\forall \alpha, \beta>0),
$$

we can prove that for any condition, $\langle\tau\rangle$ is larger than the classical traversal time $\tau_{\mathrm{cl}}$ defined by $\tau_{\mathrm{cl}} \equiv m d / \hbar \kappa$. That is, $\langle\tau\rangle \geq \tau_{\mathrm{cl}}$. As well, the imaginary part of the mean traversal time vanishes under these conditions.

On the other hand, destructive interference occurs at $\kappa d=(n+1 / 2) \pi$. Under these conditions, the mean traversal time is

$$
\langle\tau\rangle=\frac{2 m k d}{\hbar A}-i \frac{m B}{\hbar \kappa^{2} A} .
$$

In particular, we have

$$
\operatorname{Re}[\langle\tau\rangle]=\frac{2 m k d}{\hbar A} \leq \tau_{\mathrm{cl}} .
$$

Hence, the real part of $\langle\tau\rangle$ is smaller than the classical traversal time for any case of destructive resonance.

Figure 4 shows $|\langle\tau\rangle|-\tau_{\mathrm{cl}}$ as a function of $E / V_{0}$. There are several peaks around the resonance conditions. The absolute value $|\langle\tau\rangle|$ approaches the classical traversal time for larger $E$. Thus, the particle experiences a longer traversal time under resonance conditions as a result of multiple quantum reflections at the boundaries. This explains why nonuniform suppressions of transmission probabilities in the presence of dissipation occur. That is, the longer traversal time leads to larger dissipation because the damping factor $f(t)$ depends on the traversal

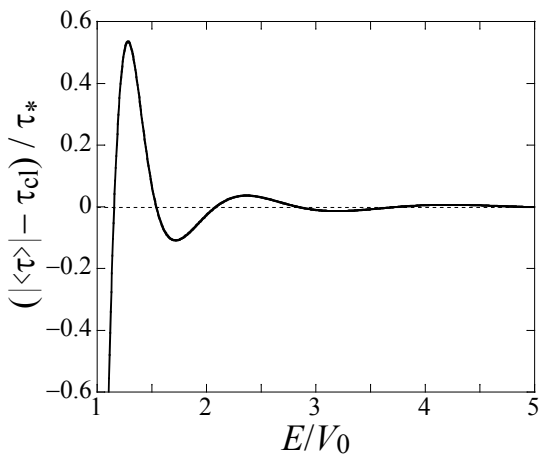

FIG. 4: Deviation of the mean traversal time from the classical traversal time, $|\langle\tau\rangle|-\tau_{\mathrm{cl}}$, in the absence of dissipation. In the calculation, we adopted $d / \lambda_{0}=5$. The resonance points are given by $E / V_{0}=1+n^{2} \pi^{2} /\left(d / \lambda_{0}\right)^{2} \simeq 1.39,2.58,4.55, \cdots$.

time through region II. The most significant reduction in the transmission probability is then expected to occur at around the first resonance resulting from the longest traversal time compared to the classical one as shown in Fig. 4 ,

This can be seen in the formula of the transmission amplitude $w_{\mathrm{D}}\left(E, V_{0}\right)$ with dissipation Eq. (18). It can be rewritten as

$$
w_{\mathrm{D}}\left(E, V_{0}\right)=w\left(E, V_{0}\right) \int_{0}^{\infty} d \tau \sqrt{f(\tau)} F(\tau) .
$$

In the absence of dissipation, we can reproduce the nondissipative expression since $\int_{0}^{\infty} d \tau F(\tau)=1$. The function $f(\tau)$ is a positive-definite decaying function as mentioned above. Hence, the factor $\sqrt{f(\tau)}$ in Eq. (29) is considered to weaken the contribution from paths with a longer traversal time through region II. Therefore, paths with a shorter traversal time mainly contribute to the probability amplitude in the presence of dissipation.

\section{B. Traversal time distribution in the presence of dissipation}

The dissipative effect on the traversal time also appears in traversal time distribution. Based on the same formulation[16], we can evaluate the traversal time distribution in the presence of dissipation by replacing $w$ with $w_{\mathrm{D}}$. The probability amplitude is then given by

$$
F_{\mathrm{D}}(\tau)=\frac{1}{w_{\mathrm{D}}\left(E, V_{0}\right)} \int_{-\infty}^{\infty} \frac{d \omega}{2 \pi} e^{-i \omega \tau} w_{\mathrm{D}}\left(E, V_{0}-\hbar \omega\right),
$$

Note that the traversal time distribution is related to the potential variation given by the right-hand side in Eq. (30), which implies an equivalence between Fig. 1(c) and Fig. 1(d). After some calculations, we obtain

$$
F_{\mathrm{D}}(\tau)=\frac{\sqrt{f(\tau)} F(\tau)}{\int_{0}^{\infty} \sqrt{f(\tau)} F(\tau) d \tau} .
$$




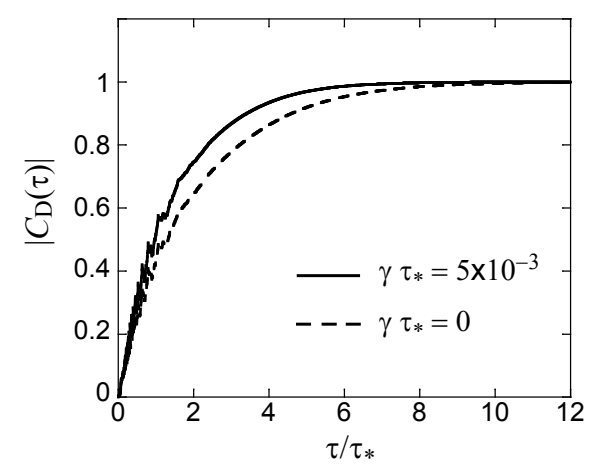

FIG. 5: The absolute value of the cumulative probability amplitude $C_{\mathrm{D}}(\tau)$. We adopted $E / V_{0}=1.3, d / \lambda_{0}=5, \Omega \tau_{*}=100$ and $\gamma \tau_{*}=5 \times 10^{-3}$ or 0 .

From the above-mentioned properties of $\sqrt{f(\tau)}$, the center of the distribution function $F_{\mathrm{D}}(\tau)$ is relatively shifted to the direction of smaller $\tau$ when compared with $F(\tau)$ without dissipation. Namely, the mean traversal time $\left\langle\tau_{\mathrm{D}}\right\rangle=\int_{0}^{\infty} \tau F_{\mathrm{D}}(\tau) d \tau$ is considered to become smaller than $\langle\tau\rangle$. This feature may be counterintuitive with respect to the idea that dissipation causes particle slow down.

This feature can be also found in the cumulative probability amplitude $C_{\mathrm{D}}(\tau)$ defined as

$$
\begin{aligned}
C_{\mathrm{D}}(\tau) & =\int_{0}^{\tau} d \tau^{\prime} F_{\mathrm{D}}\left(\tau^{\prime}\right) \\
& =\frac{1}{w_{\mathrm{D}}\left(E, V_{0}\right)} \int_{-\infty}^{\infty} d \omega \frac{\sin \omega \tau}{\pi \omega} w_{\mathrm{D}}\left(E, V_{0}-\hbar \omega(\beta 2)\right.
\end{aligned}
$$

which describes the probability amplitude for the traversal time taking a value between 0 and $\tau$. The function $C_{\mathrm{D}}(\tau)$ asymptotically approaches unity for a larger $\tau$. Figure 5 shows examples of the cumulative probability amplitude $C_{\mathrm{D}}(\tau)$ for $\gamma \neq 0$ and $\gamma=0$. Two curves converge to 1 by definition as $\tau$ becomes larger. In particular, $C_{\mathrm{D}}(\tau)$ with a non-zero $\gamma$ converges to 1 faster than the curve for $\gamma=0$. This means that the traversaltime distribution becomes narrower and the mean value becomes smaller when the dissipative effect is taken into account. This reduced mean value arises from the path selection caused by the dissipative effect, whereby paths taking a longer time in region II are effectively discarded. Therefore, the mean traversal time becomes shorter.

\section{SUMMARY AND DISCUSSION}

We have studied the effect of dissipation on the quantum transmission of a particle through a rectangular potential barrier, especially focusing on transmission resonance. We extended the path decomposition method to incorporate the dissipative effect into the calculations of the quantum transmission. The transmission probabilities are always suppressed by the effect of dissipation, especially at the first resonance because of the longer traversal time. As well, the mean traversal time in the presence of dissipation becomes smaller than that in a non-dissipative case. This is the result of path selections due to dissipation. We have not restricted ourselves to a specific scale. Thus our theory is applicable to any scale, including a macroscopic one. The study of transmission resonance of a macroscopic object is useful to test an applicability of quantum mechanics on a macroscopic scale.

Finally, we discuss an experimental setup for such a macroscopic object to test our theory. Such an experiment could be realized in a specific macroscopic system. A promising candidate is a system consisting of a fluxon in a long Josephson junction. The fluxon is a topological soliton excitation with a quantum unit of magnetic flux produced by a circulating supercurrent, i.e., a vortex, and is regarded as a single free macroscopic particle characterized by a huge number of microscopic degrees of freedom. 20] It also behaves like a quantum particle 21] in a mesoscopic Josephson junction with small capacitance per unit area. In fact, the quantum tunneling of a fluxon has recently been observed in a long annular Josephson junction [22].

Under these circumstances, a fluxon transmission experiment is possible. The potential barrier for the fluxon can be made of a microshort, 23], which is a part made of a thinner insulator than the other part. The study of fluxon transmission will complement that of quantum tunneling in research on macroscopic quantum phenomena. Moreover, a fluxon transmission experiment will also provide an important basis for implementing quantum computation in superconducting nanocircuits. Indeed, a qubit using superposition states of fluxons or breathers has recently been considered [24].

\section{Acknowledgments}

This work was supported in part by a Grant-in-Aid for Scientific Research from The 21st Century COE Program "Topological Science and Technology", by a Grant-in-Aid for Scientific Research (18540352) from the Ministry of Education Culture, Sports, Science and Technology of Japan and by JSPS KAKENHI (17740267). One author (K.K.) thanks Y. Asano for useful conversations. The numerical calculations were carried out on computers at YITP in Kyoto University.

\section{APPENDIX A: PROPAGATOR AND ITS GREEN'S FUNCTION}

In the energy representation, the propagator is expressed by the Fourier transformation as

$$
G\left(x_{T}, x_{0} ; E\right) \equiv i \int_{0}^{\infty} d T K\left(x_{T}, x_{0} ; T\right) e^{i E T / \hbar} .
$$


Equation (1) is now reexpressed in this representation as

$$
\begin{aligned}
& G\left(x_{T}, x_{0} ; E\right) \\
& =G^{(\mathrm{I})}\left(a, x_{0} ; E\right) \Sigma_{x}^{a}\left(G^{(\mathrm{II})}(b, x ; E)\right) \Sigma_{x}^{b}\left(G^{(\mathrm{III})}\left(x_{T}, x ; E\right)\right) \\
& +G^{(\mathrm{I})}\left(a, x_{0} ; E\right) \Sigma_{x}^{a}\left(G^{(\mathrm{II})}(a, x ; E)\right) \Sigma_{x}^{a}\left(G^{(\mathrm{I})}(a, x ; E)\right) \\
& \quad \times \Sigma_{x}^{a}\left(G^{(\mathrm{II})}(b, x ; E)\right) \Sigma_{x}^{b}\left(G^{(\mathrm{III})}\left(x_{T}, x ; E\right)\right)+\cdot(\mathrm{A} 2)
\end{aligned}
$$

In the case of the rectangular potential, the Green functions are given by $[16$

$$
\begin{aligned}
G^{(\mathrm{I})}\left(x^{\prime}, x\right) & =-\frac{2 m}{\hbar k} e^{-i k(x-a)} \sin k\left(x^{\prime}-a\right), \\
G^{(\mathrm{II})}\left(x^{\prime}, x\right) & =-\frac{2 m}{\hbar \kappa} \frac{\sin \kappa(x-a) \sin \kappa\left(x^{\prime}-b\right)}{\sin \kappa d} \\
G^{(\mathrm{III})}\left(x^{\prime}, x\right) & =\frac{2 m}{\hbar k} e^{i k\left(x^{\prime}-a\right)} \sin k(x-a)
\end{aligned}
$$

where $x<x^{\prime}, d \equiv b-a$ and

$$
k \equiv \frac{\sqrt{2 m E}}{\hbar}, \quad \kappa \equiv \frac{\sqrt{2 m\left(E-V_{0}\right)}}{\hbar} .
$$

Here $m$ is the particle mass. Using these Green functions, Eq. (A2) can be calculated in the form 16]

$$
G\left(x_{T}, x_{0} ; E\right)=w\left(E, V_{0}\right) G_{0}\left(x_{T}, x_{0}\right),
$$

where $G_{0}\left(x_{T}, x_{0}\right)$ is the Green function when the barrier is absent

$$
G_{0}\left(x_{T}, x_{0}\right)=-\frac{m}{i \hbar k} e^{i k\left(x_{T}-x_{0}\right)},
$$

and $w\left(E, V_{0}\right)$ is the transmission amplitude given by

$$
w\left(E, V_{0}\right)=-\frac{2 i k \kappa e^{-i k d}}{\left(k^{2}+\kappa^{2}\right) \sin \kappa d+2 i k \kappa \cos \kappa d} .
$$

The usual expression for the transmission probability is obtained as

$$
\left|w\left(E, V_{0}\right)\right|^{2}=\frac{4 k^{2} \kappa^{2}}{\left(k^{2}-\kappa^{2}\right)^{2} \sin ^{2} \kappa d+4 k^{2} \kappa^{2}} .
$$

Thus the exact transmission probability can also be obtained using the path decomposition expansion.

\section{APPENDIX B: FREE PARTICLE COUPLED TO HARMONIC OSCILLATORS}

Caldeira \& Leggett [19] published a result for the quantum Brownian motion in a harmonic potential. The result is modified in the following way for a free particle,

$$
\begin{aligned}
& J\left(x_{\mathrm{f}}, y_{\mathrm{f}}, t ; x_{\mathrm{i}}, y_{\mathrm{i}}, 0\right) \\
& =\frac{N(t)}{\pi \hbar} \exp \left[\frac { i } { \hbar } \left\{K(t)\left(\zeta_{\mathrm{f}} \xi_{\mathrm{f}}+\zeta_{\mathrm{i}} \xi_{\mathrm{i}}\right)-L(t) \zeta_{\mathrm{i}} \xi_{\mathrm{f}}\right.\right. \\
& \left.\left.\quad-N(t) \zeta_{\mathrm{f}} \xi_{\mathrm{i}}-\gamma \frac{m}{2}\left(\zeta_{\mathrm{f}} \xi_{\mathrm{f}}-\zeta_{\mathrm{i}} \xi_{\mathrm{i}}\right)\right\}\right] \\
& \times \exp \left[-\frac{1}{\hbar}\left\{A(t) \xi_{\mathrm{f}}^{2}+B(t) \xi_{\mathrm{f}} \xi_{\mathrm{i}}+C(t) \xi_{\mathrm{i}}^{2}\right\}\right]
\end{aligned}
$$

where

$$
\begin{aligned}
K(t)= & \frac{m}{2} \sigma \operatorname{coth} \sigma t, \\
L(t)= & \frac{m}{2} \frac{\sigma e^{-\gamma t}}{\sinh \sigma t}, \\
N(t)= & \frac{m}{2} \frac{\sigma e^{\gamma t}}{\sinh \sigma t}, \\
A(t)= & \frac{2 m \gamma}{\pi} \int_{0}^{\Omega} d \omega \omega \operatorname{coth} \frac{\hbar \omega}{2 k_{\mathrm{B}} T^{\prime}} \int_{0}^{t} d \tau \int_{0}^{\tau} d s \\
& \times e^{\gamma(\tau+s-2 t)} \frac{\cos \omega(\tau-s) \sinh ^{2} \sigma \sinh \sigma s}{\sinh ^{2} \sigma t},(\mathrm{~B} 5) \\
B(t)= & -\frac{2 m \gamma}{\pi} \int_{0}^{\Omega} d \omega \omega \operatorname{coth} \frac{\hbar \omega}{2 k_{\mathrm{B}} T^{\prime}} \int_{0}^{t} d \tau \int_{0}^{\tau} d s \\
& \times e^{\gamma(\tau+s-t)} \frac{\cos \omega(\tau-s)}{\sinh ^{2} \sigma t}[\sinh \sigma \tau \sinh \sigma(s-t) \\
& \left.+\sinh \sigma(\tau-t) \sinh ^{\Omega}\right] . \\
& \frac{2 m \gamma}{\pi} \int_{0}^{\Omega} d \omega \omega \operatorname{coth} \frac{\hbar \omega}{2 k_{\mathrm{B}} T^{\prime}} \int_{0}^{t} d \tau \int_{0}^{\tau} d s e^{\gamma(\tau+s)} \\
& \times \frac{\cos \omega(\tau-s) \sinh ^{2}(\tau-t) \sinh \sigma(s-t)}{\sinh ^{2} \sigma t}(\mathrm{~B} 7) \\
C(t)= &
\end{aligned}
$$

where $J\left(x_{\mathrm{f}}, y_{\mathrm{f}}, t ; x_{\mathrm{i}}, y_{\mathrm{i}}, 0\right)$ is the propagator for the density matrix $\rho(x, y, t)$ of the free particle, i.e., $\rho(x, y, t)=$ $\int d x^{\prime} d y^{\prime} J\left(x, y, t ; x^{\prime}, y^{\prime}, 0\right) \rho\left(x^{\prime}, y^{\prime}, 0\right)$. Here, $k_{\mathrm{B}}$ is the Boltzman constant, $T^{\prime}$ is the temperature, $\zeta \equiv x+y$, $\xi \equiv x-y$, and the boundary condition is given by $\zeta(0)=\zeta_{\mathrm{i}}, \zeta(t)=\zeta_{\mathrm{f}} \xi(0)=\xi_{\mathrm{i}}$ and $\xi(t)=\xi_{\mathrm{f}}$.
[1] A. J. Leggett, Prog. Theor. Phys. Suppl. 69 (1980) 80.

[2] A. O. Caldeira and A. J. Leggett, Phys. Rev. Lett. 46 (1981) 211.

[3] A. O. Caldeira and A. J. Leggett, Annals of Physics 149 (1983) 374.

[4] R. F. Voss and R. A. Webb, Phys. Rev. Lett. 47 (1981) 265.

[5] J. R. Friedman et al., Nature (London) 406 (2000) 43.

[6] C. H. van ver Wal et al., Science 290 (2000) 773.
[7] K. Hornberger et al., Phys. Rev. Lett. 90 (2003) 160401.

[8] L. Hackermüller et al., Nature (London) 427 (2004) 711.

[9] B. Lamine et al., Phys. Rev. Lett. 96 (2006) 050405.

[10] W. Cai, P. Hu, T. F. Zheng, B. Yudanin and M. Lax: Phys. Rev. B 41 (1990) 3513.

[11] R. Bruinsma and P. Bak, Phys. Rev. Lett. 56 (1986) 420.

[12] R. Bruinsma and P. M. Platzman, Phys. Rev. B 35 (1987) 4221.

[13] A. Auerbach and S. Kivelson, Nucl. Phys. B257 (1985) 
799.

[14] D. Sokolovski and L. M. Baskin, Phys. Rev. A 36 (1987) 4604; D. Sokolovski and J. N. L. Connor, ibid. 42 (1990) 6512.

[15] L. S. Schulman and R. W. Ziolkowski, in: V. Sa-Yakanit et al. (Eds.), Proceedings of the conference on Path Integrals from mev to Mev, World Scientific, Singapore, 1989, pp. 253-278.

[16] H. A. Fertig, Phys. Rev. Lett. 65 (1990) 2321; Phys. Rev. B 47 (1993) 1346.

[17] D. Bohm, Quantum Theory, Prentice-Hall, Englewood Cliffs, NJ, 1951, p. 283.

[18] L. S. Schulman, Techniques and Applications of Path In- tegration, Dover, New York, 2005.

[19] A. O. Caldeira and A. J. Leggett, Physica 121A (1983) 587; Erratum ibid. 130A (1985) 374.

[20] D. J. Bergman, E. Ben-Jacob, Y. Imry and K. Maki, Phys. Rev. A 27 (1983) 3345.

[21] T. Kato and M. Imada, J. Phys. Soc. Jpn. 65 (1996) 2963.

[22] A. Wallraff et al., Nature (London) 425 (2003) 155.

[23] Y. S. Kivshar and B. A. Malomed, Rev. Mod. Phys. 61 (1989) 763.

[24] T. Fujii et al., in: Proceedings of Foundation of Quantum Mechanics in the Light of New Technology, 2006, pp 8891. 